\title{
Müllerian duct anomaly with congenital rectovaginal fistula: A rare case presentation
}

\author{
Y Dogra, MD; S Minhas, MD; P D Marwaha, MD \\ Department of Obstetrics and Gynaecology, Kamla Nehru State Hospital for Mother and Child, Indira Gandhi Medical College, \\ Shimla, Himachal Pradesh, India
}

Corresponding author: Y Dogra (gitu_22@yahoo.com)

Pregnancy in a rudimentary horn is a rare form of ectopic gestation and associated with high maternal mortality. Unicornuate uterus with a rudimentary horn is caused by disordered fusion of Müllerian ducts during embryonic life, which can be associated with ipsilateral renal agenesis, congenital rectovaginal fistula, imperforate anus, hypospadias and other anatomical variants of cloacal dysgenesis. Despite advances in imaging techniques such as ultrasound, the diagnosis of rudimentary horn remains elusive with confirmatory diagnosis made on laparotomy. Our patient presented with an unruptured rudimentary horn pregnancy in the second trimester with a past history of surgical correction of rectovaginal fistula. Exploratory laparotomy was done and the rudimentary horn was excised. We report this case because of the very rare association of unicornuate uterus with rudimentary horn with congenital rectovaginal fistula, which forms when the Müllerian eminence opens in the dorsal segment of the endodermal cloaca.

S Afr J OG 2015;21(1):22-23. DOI:10.7196/SAJOG.746

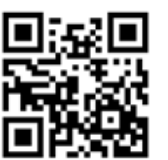

A unicornuate uterus with a rudimentary horn is a rare Müllerian abnormality that may cause many gynaecological and obstetric complications. ${ }^{[1]}$ Any disturbance in the orderly fusion of the Müllerian ducts during early embryological life (8 - 12 weeks' gestation) will result in a uterine malformation, the degree of the anomaly depending on the time the causative agent exerts its influence on the developing embryo. If the development is arrested early the uterus may remain rudimentary, and in the extreme it may even be absent. Unilateral faulty development following lack of fusion results in a uterus with one segment or one half well developed, while the other remains rudimentary or ill developed. ${ }^{[2]}$ Müllerian abnormality is frequently associated with renal and axial skeletal abnormalities. Congenital rectovaginal fistula, imperforate anus, hypospadias and other anatomical variants of cloacal dysgenesis can also be associated with maldevelopment of the Müllerian and mesonephric duct derivatives. Pregnancy in the rudimentary horn is rare and represents a form of ectopic gestation. ${ }^{[3]}$ It carries grave consequences for the mother and the fetus. ${ }^{[4,5]}$ The incidence varies from $1 / 100000$ to $1 / 150000$ pregnancies. ${ }^{[6]}$ Rupture of the pregnant rudimentary horn in the second trimester is the usual presentation, resulting in maternal morbidity and even mortality. ${ }^{[1,4]}$ Early diagnosis of rudimentary horn pregnancy (RHP) is challenging. The natural history of RHP is usually rupture during the second or third trimester, resulting in life-threatening heavy bleeding. Early prerupture diagnosis is therefore of major importance ${ }^{[5]}$ Despite advances in ultrasound prenatal diagnosis remains elusive, with confirmatory diagnosis being made at laparotomy. Because of the variable muscular constitution of the wall of the rudimentary horn, pregnancy can be accommodated until a late stage, when rupture commonly manifests as an acute abdomen with a high risk of maternal mortality. ${ }^{[3]}$

\section{Case report}

A 28 -year-old unbooked woman, gravida 3 , para $2+0$, presented to the gynaecological outpatient department at Kamla Nehru Hospital,
Shimla, Himachal Pradesh, India, with a history of amenorrhoea for 5 months and pain in the abdomen for 5 days. She brought an ultrasound report suggestive of intrauterine gestation corresponding to 20 weeks with missed abortion. She had had two previous normal vaginal deliveries at term, with an uneventful course post partum. At the age of 14 years, after menarche, she had undergone anogenital surgery; an anal opening had been created along with the correction of a rectovaginal fistula. Since then she had had normal bowel habits. On abdominal examination the height of the uterus corresponded to the period of gestation, fetal parts were palpable, and the uterus was relaxed with adequate liquor. On vaginal examination, the os was closed and the cervix uneffaced. An ultrasound scan revealed an intrauterine missed abortion. Induction was done with misoprostol $200 \mathrm{mg}, 5$ doses at 4-hourly intervals, but there was no response. On repeat vaginal examination the uterus was deviated to the right side with fullness in the left fornix corresponding to period of gestation. With a secondary abdominal pregnancy or a broad-ligament heterotopic pregnancy in mind, it was decided to perform exploratory laparotomy. On exploration, a non-communicating rudimentary horn of gravid uterus was found on the left side, with a parous-size uterus on the right (Fig. 1). Hysterotomy was done, with extraction of a $400 \mathrm{~g}$ macerated male abortus along with the placenta. No gross congenital anomaly was detected (Fig. 2), and no communication between the gravid rudimentary horn and the cervix was found. Excision of the rudimentary horn was done (Fig. 3) and haemostasis was achieved. Right-sided tubectomy was performed. The kidneys were normal.

\section{Discussion}

Unicornuate uterus with a non-communicating horn that contains menstruating endometrium is caused by asymmetrical obstruction of lateral fusion of the Müllerian ducts with ipsilateral renal agenesis. A unicornuate uterus can be present alone or with a rudimentary horn or bulb on the opposite side. Most rudimentary horns are noncommunicating. The two sides may be connected by a fibromuscular 


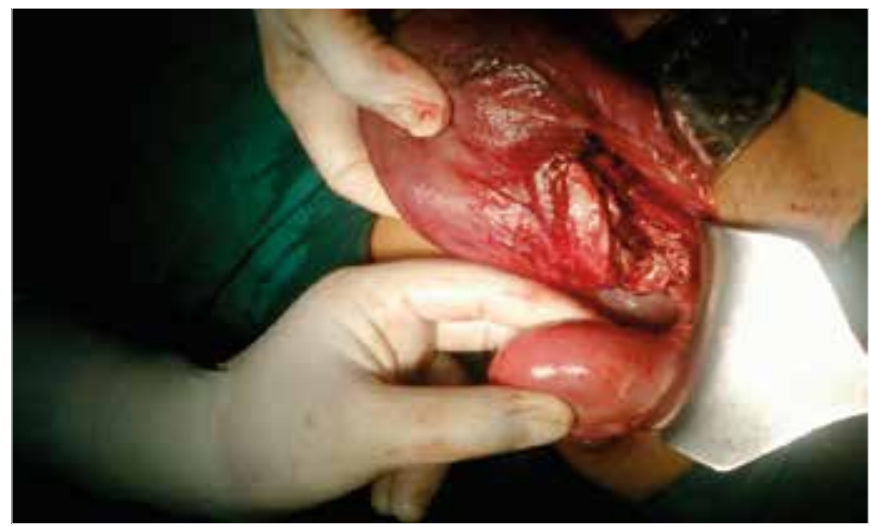

Fig. 1. Gravid rudimentary horn with unicornuate uterus attached by fibromuscular band.

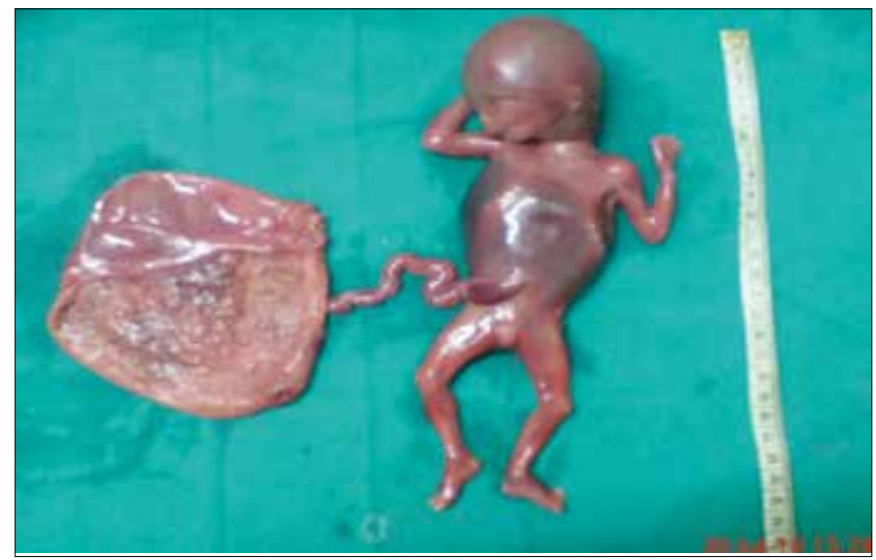

Fig. 2. Macerated male fetus.

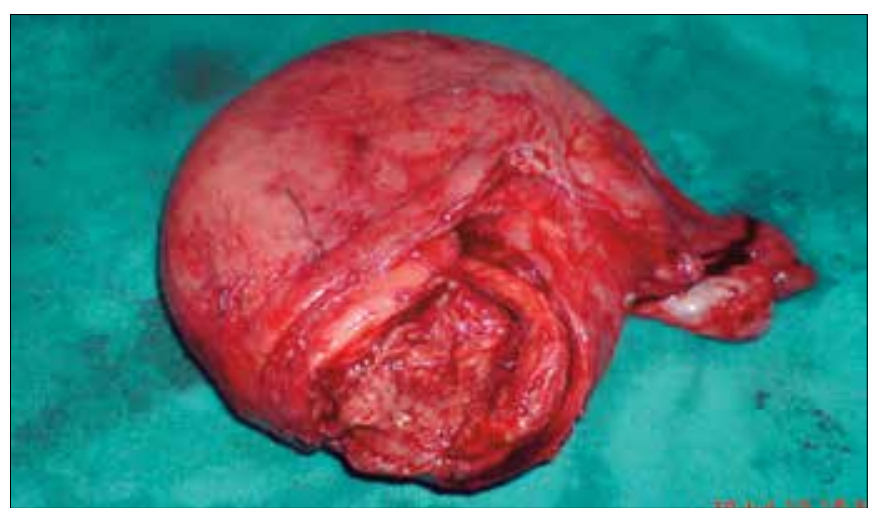

Fig. 3. Excised rudimentary horn.

band, or there may be no connection and no communication between the two uterine cavities. Because most cases of unicornuate uterus have a non-communicating rudimentary uterine horn on the opposite side, pregnancy in the rudimentary horn can result from transperitoneal migration of sperm or ovum from the opposite side. Signs and symptoms of an ectopic pregnancy develop, with eventual rupture of the horn if the pregnancy is not detected early. Rupture through the wall of the vascular rudimentary horn is associated with sudden and severe intraperitoneal haemorrhage and shock. Death can occur in a few minutes. ${ }^{[7]}$ Congenital rectovaginal fistula, imperforate anus, hypospadias and other anatomical variants of cloacal dysgenesis can also be associated with maldevelopment of the Müllerian and mesonephric duct derivatives. ${ }^{[8]}$ Rudimentary horn with congenital rectovaginal fistula forms when Müllerian eminences open in the dorsal segment of the endodermal cloaca. ${ }^{[9]}$ Unicornuate uterus with noncommunicating rudimentary horn is a rare condition but is associated with many gynaecological and reproductive morbidities. A 7-year study conducted by Goel et al. ${ }^{[10]}$ showed that preoperative diagnosis of noncommunicating RHP was possible in 2 out of 18 cases of unicornuate uterus with non-communicating rudimentary horn found on laparotomy. Pregnancies in women with this condition were associated with high incidences of abortion, preterm labour, malpresentations and caesarean delivery. ${ }^{[10]} \mathrm{A}$ retrospective study ${ }^{[11]}$ on 42 women with a unicornuate uterus and rudimentary horn was undertaken in a university hospital in Finland. The rudimentary horn was removed in 21 cases. Right unicornuate uterus with a non-communicating rudimentary horn was the most common type of uterine anomaly. Unilateral renal agenesis was found in $38 \%$ of cases and ectopic pregnancy occurred in $22 \%$. The pregnant uterine horn ruptured in 3 of 7 cases. The high number of ectopic pregnancies indicates removal of rudimentary horn and its tube when diagnosed. ${ }^{[1]}$ The availability of technological advances such as ultrasonography and magnetic resonance imaging (MRI) has made the diagnosis of RHP possible at an early stage of gestation. However, in advanced pregnancy such cases can sometimes pose a diagnostic dilemma and are recognised only when the patient presents with abdominal pain and collapse and is taken for laparotomy. ${ }^{[12]}$ Tsafrir et al ${ }^{[13]}$ suggested the following criteria for early sonographic diagnosis of rudimentary horn pregnancy: (i) a pseudopattern of asymmetrical bicornuate uterus; (ii) absent visual continuity tissue surrounding the gestation sac and the uterine cervix; and (iii) presence of myometrial tissue surrounding the gestational sac. MRI has proved to be a very useful, non-invasive tool for diagnosis of Müllerian abnormalities. It offers multiplanar images without the hazards of ionising radiation and is able to show both internal and external uterine structures. ${ }^{[13]}$

\section{Conclusion}

Detailed clinical evaluation with a high degree of suspicion is required in patients presenting with an acute abdomen, especially when other Müllerian/mesonephric duct abnormalities are present. Every effort should be made to diagnose RHP in time to prevent rupture, which may be associated with high maternal mortality. If diagnosed on laparotomy, the excision of the rudimentary horn with ipsilateral tubectomy should be undertaken.

1. Chopra S, Suri V, Aggarwal N. Rudimentary horn pregnancy: Prerupture diagnosis and management. Indian J Med Sci 2007;61(1):28-29. [http://dx.doi.org/10.4103/0019-5359.29595]

2. De Silve PHDH. Rudimentary horn of a bicornuate uterus: Discussion of 16 cases with a review of literature. J National Sci Coun Sri Lanka 1976;4(1):55-73.

3. Elsayegh A, Nwosu EC. Rupture of pregnancy in the communicating rudimentary horn at 34 weeks. Hum Reprod 1998;13(12):3566-3568. [http://dx.doi.org/10.1093/humrep/13.12.3566]

4. Dhananjaya BS, Shobha UN, Nanda SK, Nandagopal KM, Anitha MS. A rare case of pregnancy in the rudimentary horn of unicornuate uterus (on table diagnosis) which had a successful outcome A case report. Journal of Clinical and Diagnostic Research 2011;5(7, Suppl 2):1461-1463.

5. Nagarathna G, Navada HM, Poornima B, Bhat R. Pre-rupture diagnosis and management of rudimentary horn pregnancy in second trimester. Int J Pharm Biomed Res 2011;2(3):179-181.

6. Ural SH, Artal R. Third-trimester rudimentary horn pregnancy. A case report. J Reprod Med 1998;43(10):919-921.

7. Edmonds DK. Dewhurst's Textbook of Obstetrics \& Gynaecology. 7th ed. Hobokon, NJ: Blackwell, 2007:331. 8. Rock JA, Jones HW. Te Linde’s Operative Gynecology. 10th ed. Philadelphia: Lippincott Williams \& Wilkins, 2008:539-544, 574-575.

9. Jacob A. A Comprehensive Textbook of Midwifery \& Gynecological Nursing. 3rd ed. New Delhi: Jaypee, 2012:702-709.

10. Goel P, Aggarwal A, Devi K, Takkar N, Saha PK, Huria A. Unicornuate uterus with noncommunicating rudimentary horn - different clinical presentations. J Obstet Gynecol India 2005 55(2):155-158.

11. Heinonen PK. Unicornuate uterus and rudimentary horn. Fertil Steril 1997;68(2):224-230. [http:// dx.doi.org/10.1016/S0015-0282(97)81506-3]

12. Jain R, Gami N, Puri M, Trivedi SS. A rare case of intact rudimentary horn pregnancy presenting as hemoperitoneum. J Hum Reprod Sci 2010;3(3):113-115. [http://dx.doi.org/10.4103/0974-1208.69335]

13. Tsafrir A, Rojansky N, Yitzhak Sela H, Gomori JM, Nadjari M. Rudimentary horn pregnancy: Firsttrimester prerupture sonographic diagnosis and confirmation by magnetic resonance imaging. Ultrasound Med 2005;24(2):219-223 